\title{
Front propagation dynamics: Qualitative differences revealed by very high intensity fluctuations
}

\author{
Sergio E. Mangioni" and Javier Fernandez Acevedo \\ IFIMAR (Universidad Nacional de Mar del Plata and CONICET) and Deán Funes 3350, B7602AYL Mar del Plata, Argentina
}

(Received 6 April 2018; revised manuscript received 26 September 2018; published 14 November 2018)

\begin{abstract}
This paper presents a detailed analysis of the effect of a large external noise on the propagation of a front between two stable steady states. Noise affects one of the steady states or the thresholds. Significant differences with respect to the case of small noise intensity are carefully studied using a Langevin approach. The average effect of noise is modeled by means of an additional nonlinear term that enables an analysis of short-time and long-time behaviors. Nontrivial behaviors are numerically observed and analytically discussed: (i) Two symmetry breakings of the fluctuation-free stable states are observed and (ii) two fronts propagating in opposite directions are simultaneously observed and the propagation direction may be exchanged by changing the diffusion coefficient.
\end{abstract}

DOI: 10.1103/PhysRevE.98.052213

\section{INTRODUCTION}

Propagating fronts into metastable states or phases is a well-known phenomenon [1]. It is the most effective way for a phase transition to take place between a stable state and another metastable one. According to Maxwell's area law, both phases coexist (and the front remains at rest) when the areas corresponding to each attraction basin are equal (Maxwell's point). Then, when these areas are unbalanced, the front moves into the (metastable) least-area phase with a velocity proportional to the gap between such areas, with the greatest-area phase (the one with least potential) remaining. A front velocity reversion is only produced by varying some parameter characterizing the nonlinearity in a way that yields a reversion of the area relation. A statistical description is required when the system is affected by additive noise. However, the deterministic states coincide with the most probable values and the area relation does not change in comparison to the noiseless case, with the mean propagation velocity of the front equal to the deterministic one [2,3].

The situation is different when the noise is multiplicative. For a given generic nonlinearity $F_{0}(u)$ and multiplicative factor $\Gamma^{1 / 2}(u)$ the stochastic equation governing the dynamics is

$$
\partial_{t} u=F_{0}(u)+D \partial_{x}^{2} u+\Gamma^{1 / 2}(u) \eta(x, t),
$$

where $D$ is the diffusion coefficient and $\eta(x, t)$ a zero-mean Gaussian white noise characterized by

$$
\langle\eta(x, t) \eta(x, t)\rangle=\bar{\lambda}^{2} \delta\left(x-x^{\prime}\right) \delta\left(t-t^{\prime}\right) .
$$

An analysis of the long-time average dynamics shows that noise tends to destabilize the noisier state in favor of the noiseless one $[4,5]$. In such a case, the corresponding average dynamics can be described by an equation as (see Appendix):

$$
\partial_{t} u=F_{0}(u)-\frac{\sigma^{2}}{2} \Gamma^{1 / 2}(u) \partial_{u} \Gamma^{1 / 2}(u)+D \partial_{x}^{2} u,
$$

*smangio@mdp.edu.ar where $\sigma^{2}:=\bar{\lambda}^{2} \delta x$, where $\delta x$ is a (dimensionless) cutoff at short length scales of the $x$ domain. By using results describing the so-called entropic mechanism [6,7], a similar equation was obtained to study constructive effects of noise in a system with aggregating current (attractive lateral interactions) [8]. We note that an effective nonlinearity describing the corresponding average dynamics arises, with the noise average effect considered in a term like Stratonovich's which is subtracted to deterministic nonlinearity [5]. Engel obtained this same equation, in a different way, to study front propagation, resulting in a multiplicative noise that drives the front into the noisier phase, with a mean propagation velocity proportional to the gap between the squared noise multiplicative factor, evaluated at each phase connected by the front (result validated for low noise intensity) [2]. This result enables us to apply a generalization of the Maxwell's area law by replacing the $F_{0}(u)$ role by the above-mentioned effective nonlinearity.

A widespread proposal to describe front behavior is to consider the noise effect as separated into a systematic part and a fluctuating part. The systematic part, consisting of the mean value of noise term and equivalent to Stratonovich's term, is added to the nonlinearity as a deterministic effect, and thus the sum of both terms constitute an effective nonlinearity describing the average front behavior. On the other hand, the fluctuating part is built subtracting the mean values from the noise term, and therefore this does not contribute to the mean displacement of the front since its average is null $[3,9,10]$. In short, a zero is added to the stochastic equation by adding and subtracting a term like Stratonovich's. Therefore, the equation governing the corresponding average dynamics - deterministic nonlinearity plus the systematic part (noise term) equals diffusive propagation-is written as:

$$
\partial_{t} u=F_{0}(u)+\frac{\sigma^{2}}{2} \Gamma^{1 / 2}(u) \partial_{u} \Gamma^{1 / 2}(u)+D \partial_{x}^{2} u .
$$

This equation is suitable for describing front propagation provided that a temporal coarse-graining enables us to decouple fast fluctuations from slow ones. This in turn provides an 
averaged front shape different from the deterministic one and therefore having different velocity. By defining an effective potential from the deterministic nonlinearity added to the noise term (integrating the effective nonlinearity and changing its sign), we observe that fluctuations tend to stabilize the noisier state. Moreover, the front moves in a way that such a state is the one remaining when the deterministic nonlinearity is symmetric (Maxwell's point). In short, according to this second approach, the average effect of fluctuations promotes the selection of the noisier state by means of the front propagation. We emphasize that this result is opposite of that obtained using Eq. (3), which at Maxwell's point predicts the stabilization of the noiseless state in the long timescale. By considering that both equations describe the average front behavior modeling the multiplicative noise effect by means of an extra term like that of Stratonovich's but with opposite sign, the fact that these equations predict front velocities with opposite directions should not be surprising. We note that while the effective nonlinearity corresponding to the first approach is the same one used to describe long-time behavior, its equivalent (deterministic nonlinearity plus the systematic part) corresponding to the second approach is the same one used to calculate the known approximation describing shorttime behavior [4,5]. This suggests that if the front velocity is low enough, the first approach [Eq. (3)] would be the appropiate one, since a front that is slow enough should connect phases corresponding to long-time behavior. Such a situation can be expected for high noise intensity, but in that case it can also be expected that the chance for a front to be stabilized is very low, since noise would far overtake any threshold. By contrast, for a bit higher front velocity (although not high enough to overcome fluctuations) it would correspond to applying the second approach [11]. However, when comparing the dynamics corresponding to the long and short timescales we observe that although a front could drive the system toward the noisier state in the short timescale, it could also happen that in the long timescale the same system evolves from the noisier state toward the less-noisy state.

In general, researchers resorted to the second approach, obtaining results that were consistent with numerical simulations $[3,9,10]$. We note that the second approach was initially validated for low-enough noise intensity (small-noise expansion approach) [3,9], but Novikov's theorem used in the context of front propagation in reaction-diffusion systems enables this result to be applied for high noise intensity, since this approach does not necessarily involve a series expansion in powers of noise intensity nor any other approximation [10]. Despite the qualification of Novikov's theorem, the studies about front propagation driven by multiplicative noise were not focused on situations of high noise intensity $[3,9,10]$.

It is known that natural systems are undeniably subject to random fluctuations, arising from either environmental variability or thermal effects. Due to inherent complexity, an environment parameter is typically subject to a complex web of diverse processes, varying at different scales, both in space and time. Therefore, it would be more realistic to model its complicated behavior by means of a stochastic variable [12]. Thus, for example, numerous reports emphasizing the relevancy of environmental fluctuations effects on biological systems have motivated interesting results about constructive effects driven by mesoscopic parameter fluctuations describing a given system $[12,13]$. The range of noise intensity is wide and therefore not necessarily limited to low values. Front propagation in a bistable system does not escape to this reality.

In this paper we consider cases with high noise intensity. We particularly studied an extended system governed by a cubic nonlinearity, with one of its roots affected by fluctuations. This proposal gives birth to a multiplicative noise with a quadratic factor having two roots, and, as a consequence, the noise is null when the field matches such roots. Propagating fronts subject to the above conditions have already been studied through the second approach but for situations of low noise intensity $[3,10]$. The results notably change when noise intensity is high. For the zero-dimensional case, strongenough fluctuations of one of the stable states can induce an exchange of roles between the fluctuating state and the unstable (threshold) state [14]. We note that this is a phenomenon appreciable at a long timescale, which is why this may not impact front behavior. However, when a diffusion term is incorporated, two spatial symmetry breakings arise as a result of cooperation between fluctuations and spatial coupling: The two stable states become unstable and are replaced by four new ones (two per symmetry-breaking) [15]. In this paper we show evidence that these symmetry breakings arise from a short-time instability reinforced by spatial coupling, like in Refs. [4,5].

The above-mentioned phenomenon remains hidden for low noise intensity for two reasons: (a) There is a critical noise intensity beyond which the symmetry breakings are produced and (b) even if noise intensity exceeds the critical value (or threshold), the emerging twin states at each destabilized state are too close to each other for them to be easily detected, unless the intensity is substantially increased. Therefore, on average, the propagating fronts subject to the conditions just mentioned behave as if they were governed by an effective nonlinearity given by the sum of the deterministic nonlinearity and the systematic fluctuating part (term indicated like the Stratonovich's one). So, for low noise intensity, the effective nonlinearity is what sets the average front velocity. However, when the intensity is high, both symmetry breakings become evident and they strongly impact front propagation, as we will show here. In fact, as noise intensity increases the two emerging twin states separate more and more until the two states (not twins) located in the field central region (between the two states destabilized) overlap, establishing three stable states instead of four [15].

In this paper we will show numerical simulation results that reveal an unusual behavior in front propagation dynamics under a high-noise-intensity regime. We will propose strong fluctuations of one of the deterministic stable solutions as well as the threshold. We will present also the corresponding probability distribution in order to highlight the variation of the new mean phases and their relative stability as the coupling constant changes. Next, we will show propagating fronts connecting noisy phases, each one confined within one of three possible regions, highlighting the field value dispersion within each region. We will also show how the front velocity is reversed as the coupling constant changes, which is inconsistent with the behavior observed for low noise intensity. Finally, after calculating the SPDFs and relating 
them to the front velocity direction, we uphold the thesis that this phenomenon is linked to the long-time system behavior. The paper structure is as follows: description of the model and phenomenon, numerical results, analysis, and conclusions.

\section{MODEL AND DESCRIPTION OF PHENOMENON}

In order to describe the system, we define a dimensionless $u$ field varying between zero and 1. For example: If the model describes population dynamics, by considering each individual requires a minimum vital space (a space that cannot be invaded by another individual) and by definining $u$ as the covering degree of the available space, $u$ can be taken as dimensionless and normalized to 1 . For the case in which the field describes substance content (for example, phosphorus in a model of lake eutrophication [16]), its maximum content can be used to normalize $u$. Finally, $u$ can also be dimensionless and normalized to 1 when describing the covering degree of an adsorbed surface.

We chose to make more visible the role of the parameters characterizing the system by expressing the aforementioned cubic nonlinearity in terms of their roots:

$$
F(u)=\left(u-\beta_{d}\right)(u-\alpha)\left(\beta_{u}-u\right) .
$$

Hence, our control parameters are the nonlinearity roots. The two uniform attractors are $u=\beta_{d}$ and $u=\beta_{u}$ and the ejector (threshold) is $\alpha$ (separating both attraction basins). We introduce fluctuations to each parameter by adding to its mean value a zero-mean Gaussian white noise $\eta(x, t)$, with a correlation given by Eq. (2). Thus, fluctuations around the mean value of any of these parameters lead to multiplicative noises with a factor which is quadratically dependent on the field as $\Gamma^{1 / 2}(u)=(u-\alpha)\left(u-\beta_{u}\right)$ for $\beta_{d}$ fluctuations and $\Gamma^{1 / 2}(u)=\left(u-\beta_{d}\right)\left(u-\beta_{u}\right)$ for $\alpha$ fluctuations.

As a significant fact for our results, we remark that both multiplicative factors are zero, provided that the field values match any of the two roots unaffected by fluctuations (in other words, fluctuation-free roots).

Thus, the corresponding stochastic dynamics is described by Eq. (1), where now the subindex 0 in $F_{0}(u)$ indicates $F(u)$ but replacing the fluctuating parameter $(\beta$ or $\alpha)$ by its mean value $\left(\beta_{d 0}\right.$ or $\left.\alpha_{0}\right)$. We note that Eq. (1) is to be interpreted in the Stratonovich's sense.

The noise multiplicative-factor roots separate the field variability range into three regions, which we denote as I, II, and III. Their definitions depend on which parameter is the one that fluctuates, which we now outline as follows: when $\beta_{d}$ fluctuates, region I is between $u=0$ and $u=\alpha$, region II is between $u=\alpha$ and $u=\beta_{u}$, and region III is between $u=\beta_{u}$ and $u=1$. When $\alpha$ fluctuates, region $\mathrm{I}$ is between $u=0$ and $u=\beta_{d}$, region II is between $u=\beta_{d}$ and $u=\beta_{u}$, and region III is between $u=\beta_{u}$ and $u=1$. For high noise intensity, numerical simulations of Eq. (1) show that when taking an initial field profile confined to one of these regions, the system evolves in a way that the field always remains within such a region [15]. As a consequence, we consider the frontiers between regions to be "impassable walls" for the field, provided that such regions are not connected by field gradients. The same above behavior is observed for low noise intensity when $\alpha$ fluctuates, but there is a difference

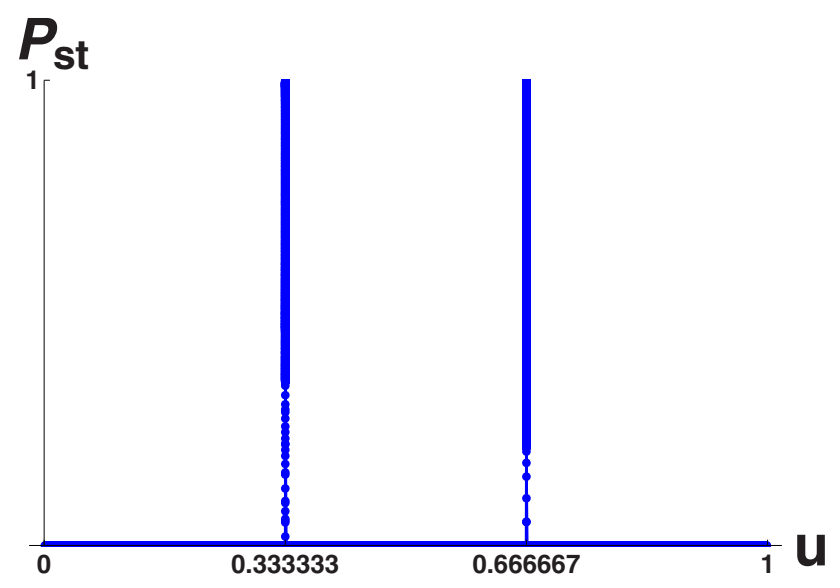

(a)

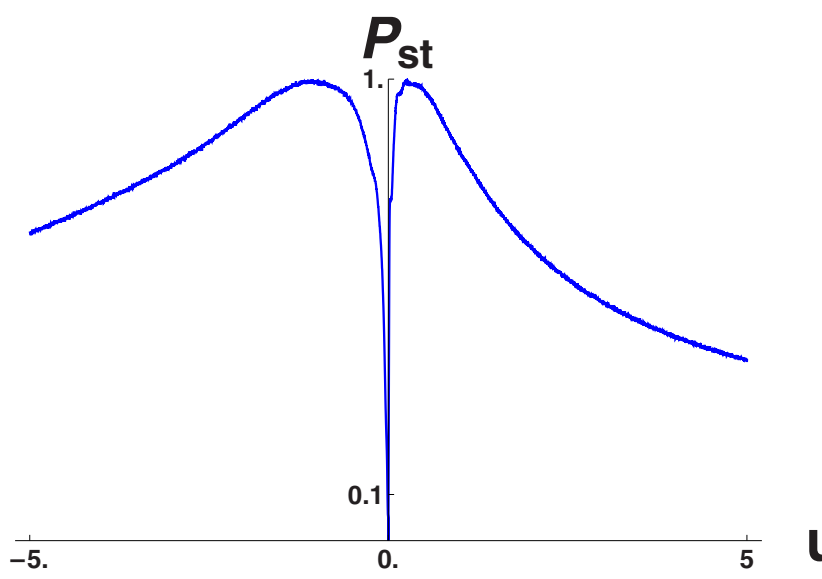

(b)

FIG. 1. SPDF vs. field for $\alpha$ fluctuations around $\alpha_{0}=0.5$, with $\beta_{d}=0.333333, \beta_{u}=0.6666667, \lambda=1.5$, and $K_{a}=3.4$. (a) $\mapsto$ overall view. (b) $\mapsto$ zoomed image around the $u=\beta_{d}$ redefining $u$ as $u=10^{8}\left(u-\beta_{d}\right)$. Zoomed image around the $u=\beta_{u}$ is similar to the former. We note that the SPDF in $u=\beta_{d}$ and $u=\beta_{u}$ is zero.

in behavior when it is $\beta_{d}$ the one that fluctuates: The $\alpha$ root does not work as an impassable wall. We note that in such case while $u=\alpha$ is always a root of $F_{0}^{\mathrm{eff}}(u)$, provided that the system is zero dimensional, for low noise intensity $u=\alpha$ is an ejector and for high noise intensity $u=\alpha$ is an attractor [14]. Therefore, the noise multiplicative factor roots only work as "impassable walls" when they are attractors of the zero-dimensional system.

Both numerical simulations calculating the stationary probability density function [SPDF obtained from Eq. (1)], as well as the SPDF obtained from Eq. (3) - the one that governs the long-time dynamics - by means of a mean-field approximation, show symmetry breaking in each fluctuationfree stable root [15]. Although it is hard to detect, such a phenomenon occurs even when noise intensity is low. Figure 1 shows the SPDF vs. field curve for a low noise intensity (corresponding to $\alpha$ fluctuating, coupling constant $K_{a}=\frac{2 D}{\delta x^{2}}$, and $\lambda=1.5$, with $\lambda=\frac{\sigma}{\beta_{u}}$; the parameter we choose to set the noise intensity value). In order to focus on the effects of the noise and coupling, we minimize the deterministic force driving the 
front by selecting parameter values that set a quasisymmetric deterministic nonlinearity. Here the curves were numerically calculated for each region separately (see Ref. [15] for the calculation method) and then they were combined into the same curve and normalized (using a fictitious criterium to enhance the curve visibility). Since the field values do not cross limits between regions, curve heights calculated this way are not comparable between regions. These were thus calculated only to highlight all the possible phases that might be connected by fronts (including the metastable ones). We note that the averages were calculated using 30 different realizations (each one initiated from a different seed). The top curve in Fig. 1 appears to show only two phases that match the fluctuation-free roots, but such as it is shown in bottom curve in Fig. 1, by expanding the image around of the each peak, both symmetry breakings (twin states) are revealed.

As previously mentioned, there is a critical noise intensity from which the symmetry breakings occur. We note that the aforementioned mean-field approximation, which was implemented from an equation describing a long-time dynamics and supports the numerically observed symmetry breakings [15], does not enable us to detect such a critical point. We consider that the reason for this is that the instability causing such a phenomenon appears in the short timescale. In order to prove this we calculate the short-time evolution of the first moment of the probability density as in Ref. [5] after applying a mean-field approximation that causes the elimination of the diffusion term. Under these conditions, the equation governing the evolution of the first moment can be written as:

$$
\partial_{t}\langle u\rangle=F_{0}(\langle u\rangle)+\bar{\lambda}^{2} \partial_{\langle u\rangle} \Gamma(\langle u\rangle),
$$

where $\langle u\rangle$ is the first moment of $u$. A simple calculation using Eq. (6) enables us to show that there is a short-time instability per each fluctuation-free stable root. This short-time instability, which is induced by the multiplicative noise and reinforced by spatial coupling, both acting in cooperation, is the one producing each one of symmetry breakings observed. Given that, when close to the critical point, the most likely field values are near the fluctuation free roots, a linearization of both terms is applicable. For $\alpha$ fluctuations and around $u=\beta_{u}$ we obtain:

$$
\partial_{t}\langle u\rangle=\left[\left(\beta_{u}-\beta_{d}\right)^{2} \frac{\bar{\lambda}^{2}}{2}-\left(\beta_{u}-\beta_{d}\right)\left(\beta_{u}-\alpha_{0}\right)\right]\langle u\rangle,
$$

and, therefore, the critical noise intensity is $\bar{\lambda}_{c}=2 \sqrt{\frac{\beta_{u}-\alpha_{0}}{\beta_{u}-\beta_{d}}}$. Following the same procedure, for $\alpha$ fluctuations and around $u=\beta_{d}$ we obtain $\bar{\lambda}_{c}=2 \sqrt{\frac{\alpha_{0}-\beta_{d}}{\beta_{u}-\beta_{d}}}$, and, finally, for $\beta_{d}$ fluctuations and around $u=\beta_{u}$ we obtain $\bar{\lambda}_{c}=2 \sqrt{\frac{\beta_{u}-\beta_{d 0}}{\beta_{u}-\alpha}}$.

The issue is more complex for $\beta_{d}$ fluctuations and around $u=\alpha$. The multiplicative noise produces an effective nonlinearity that shifts the root $\beta_{d}$ toward values that go beyond $\alpha$. This is a long-time phenomenon that can be described by an equation such as Eq. (3) but without the diffusion term [14]. Therefore an exchange of roles happens and now $\alpha$ is homogenously stable while the threshold is an effective $\beta_{d}^{e}>\alpha$ which is obtained by adding a noise term to the original one. When noise intensity is enough to produce such an exchange of roles, $\alpha$ becomes unstable in the short-time

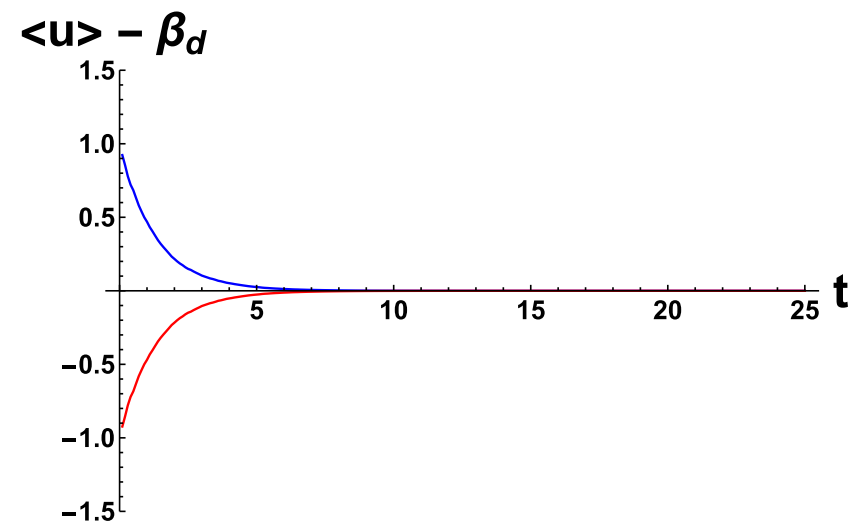

(a)

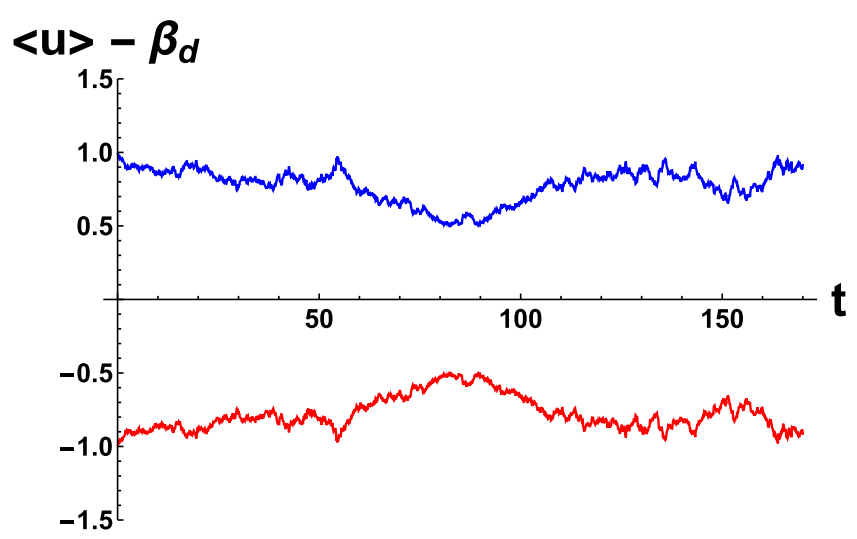

(b)

FIG. 2. Field average value $\left(\langle u\rangle-\beta_{d}\right)$ relative to $\beta_{d}$ vs. time considering two cases: one below and the other over $\lambda_{c}$ for fluctuations of $\alpha$ around $\alpha_{0}=0.5$, with $\beta_{d}=0.333333, \beta_{u}=0.6666667$, and $K_{a}=10$. (a) The case for $\lambda=1.4$ when initiating the system from the top curve $\mapsto u=\beta_{d}+10^{-7}$ and the bottom curve $\mapsto u=$ $\beta_{d}-10^{-7}$. Both evolutions end up in $\beta_{d}$. (b) The case for $\lambda=1.5$ when initiating the system as before. Clearly $\langle u\rangle$ fluctuates around a value different of $\beta_{d}$ for both evolutions, one at each side of $\beta_{d}$, showing the corresponding symmetry breaking. For these parameter values the critical noise intensity is between $\lambda=1.4$ and $\lambda=1.5$, which is of the order of our theoretical calculation. In order to improve the graphic, the relative field average value is set multiplying by $10^{7}$ and $t$ is set in millions of time steps.

and the symmetry-breaking occurs because of the coupling. We could believe that for solving this situation the same term is added and subtracted [see Eqs. (3) and (6)] but we must take into account that both terms act on different timescales. This is a rare situation in which $\alpha$ turns into an attractor in the long timescale by effect of the multiplicative noise, having previously been an ejector in the short timescale because of the same noise. To summarize, for this case, the critical noise intensity is the same that determines such exchange of roles.

In order to illustrate this phenomenon we show an example highlighting the transition around one of the fluctuation-free roots. Figure 2 shows a case for two noise-intensity values, one slightly lower and other slightly higher than the critical noise intensity. The evolution curves of the $\langle u\rangle$ were obtained by numerically resolving the dynamics described by Eq. (1) 


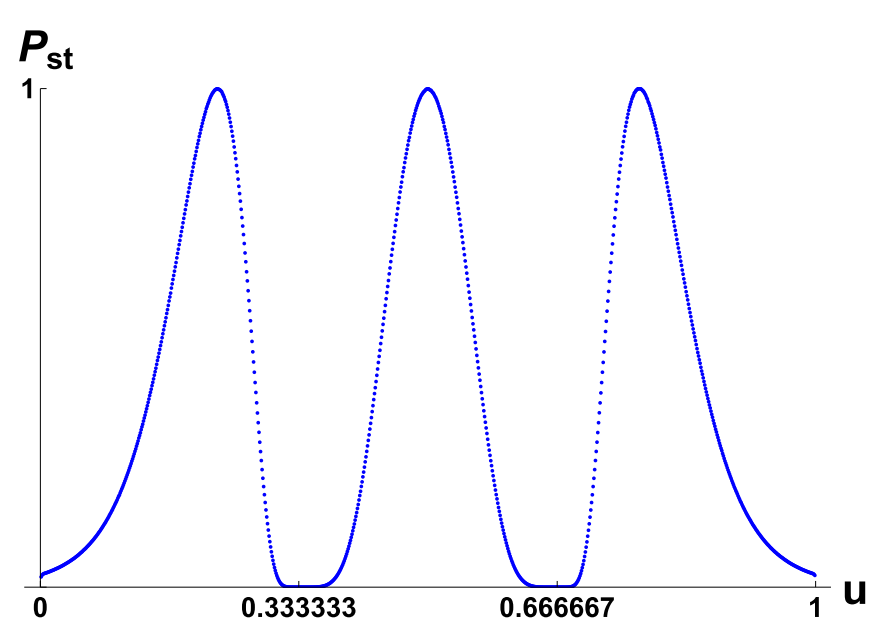

FIG. 3. SPDF vs. field for $\alpha$ fluctuations around $\alpha_{0}=0.5$, with $\beta_{d}=0.333333, \beta_{u}=0.6666667, \lambda=1.7$, and $K_{a}=3.4$.

(see details below) and by spatially averaging the field $(u)$ values along the profile. The upper graphic shows curves with $\lambda \lesssim \lambda_{c}$ as the system initiated from field values in the neighborhood of the root but from different regions. The lower graphic shows curves with $\lambda \gtrsim \lambda_{c}$ as the system initiated from the same conditions as before. It can be observed that the field asymptotically decays to the fluctuation-free root in the first case but it does not in the second one. When the critical noise intensity is overcome, the field asymptotically approaches values that differ from such root, depending on the region from which the system was initiated. We note that on no occasion does the field reach the root value or cross it.

When the noise intensity is higher, the central region peaks are merged into a unique broader peak localized in the center of such a region. Figure 3 illustrates this phenomenon by exhibiting the SPDF vs. the field for the same case as in Fig. 1 but with $\lambda=1.7$ instead of $\lambda=1.5$. The transition occurs within a small noise intensity range. In this paper we worked preferably with parameter values setting this last situation: one probability peak per region. We note that this same transition, which drives from four probability peaks to three, is also observed when the coupling $\left(K_{a}\right)$ increases. Even for quite high noise intensity, our numerical calculations show that for small-enough $K_{a}$ values there are two twin states surrounding each fluctuation-free roots.

In order to numerically resolve the stochastic dynamics, we proceed to do a spatial discretization in a regular onedimensional lattice composed of 14400 sites with spacing $\delta x=2.510^{-2}$ and time step $\delta t=10^{-4}$, i.e., $u\left(x_{i}\right) \longrightarrow u_{i}$, with $i$ the cell index. Considering that $\partial_{x}^{2} u \longrightarrow \frac{1}{\delta x^{2}} \sum_{j}\left(u_{j}-\right.$ $u_{i}$ ) (one dimension), where the sum is over the nearest neighbors, the discretized version of Eq. (3) is written as

$$
\partial_{t} u_{i}=F_{o}\left(u_{i}\right)+\frac{K_{a}}{2} \sum_{j}\left(u_{j}-u_{i}\right)+\Gamma^{1 / 2}\left(u_{i}\right) \eta_{i}(t)
$$

where $K_{a}=\frac{2 D}{\delta x^{2}}$ and now the correlation is $\left\langle\eta_{j}(t) \eta_{j}\left(t^{\prime}\right)\right\rangle=$ $\sigma^{2} \delta\left(t-t^{\prime}\right) \delta_{i j}$, with $\lambda=\frac{\sigma}{\beta_{u}}$ our reference parameter to set the noise intensity. We resolved the stochastic equation using Heun's method; although in order to obtain propagating fronts, the initial condition is a step type profile that takes uniform field values in two different regions connected by the front (in order to check result independence regarding initial conditions we also used a uniform random distribution to assign field values within each region connected by the front). As usual for stabilizing fronts, we use as boundary conditions $\partial_{x} u=0$. All averages were calculated using 30 different realizations (each one initiated from a different seed). Although the front plots correspond to a single realization, no qualitative changes arise using a different seed.

Since the unusual behavior of propagating front is revealed under the high-noise-intensity regime by varying $K_{a}$, we focus on the influence of such a constant in the front behavior. First, we explored a range of $\lambda$ values that includes the transition point between a situation in which the twin states do not exist (below $\lambda_{c}$ ) or their effects are hard to detect (slightly above $\lambda_{c}$ ). Then we considered another intensity range in which the effects of the symmetry breakings are appreciable. Whenever $\lambda<\lambda_{c}$ we observed the typical behavior: Around Maxwell's point, the fronts promote the noisier state because of noise effects, and both the front velocity and width increasing in proportion to $K_{a}^{1 / 2}$. Details related to the fact that the field does not cross the fluctuation-free root unless a field gradient between regions exists are presented in the Supplemental Material [17]. For $\lambda \gtrsim \lambda_{c}$ we recorded that while the front propagates toward the usual direction, effects of the twin states emerged within the same front (see Supplemental Material at [17]). We consider it a transition stage between the already-known behavior and the behavior we will show below.

The situation we focus on is the one characterized by one probability peak per region, such as the one shown in Fig. 3. We initiate the system from hyperbolic-tangent-like profiles connecting field values belonging to different regions. So we define initial profiles with "phases" in regions: (a) I and III, (b) I and IIu (II, choosing a field value next to $\beta_{u}$ ) and (c) IId (II, choosing a field value next to $\beta_{d}$ ), and III. Figure 4 displays the results for $\lambda=1.7$ and $K_{a}=1$ and Fig. 5 for $\lambda=1.7$ and $K_{a}=5$ (in all figures showing fronts, $x$ is set in terms of the lattice sites and $t$ in millions of time steps). A meaningful change in the nature of the front can be observed. When initiating the system from regions I-III [case (a)] with $K_{a}=1$, two fronts are stabilized and propagate with opposite velocities in a way that the dominating phase is the one from region II. These are fronts connecting phases I-II and II-III. In the same situation but with $K_{a}=$ 5 , such a phenomenon does not take place; instead, a front connecting the phases I-III stabilizes, as its velocity is much lower than the one for previous fronts. When initiating the system from regions I-II [case (b)] for $K_{a}=1$ a front I-II is stabilized and propagates with negative velocity (dominating phase: II), while for $K_{a}=5$ the front I-II propagates with positive velocity (dominating phase: I). Conversely, when initiating the system from regions II-III [case (c)] for $K_{a}=$ 1 , a front II-III is stabilized and propagates with positive velocity (dominating phase: II), while for $K_{a}=5$ the front II-III propagates with negative velocity (dominating phase: III). This is consistent with the fact that for smaller $K_{a}$ values, phase II is the most stable one (dominating), but for the largest $K_{a}$ values, phases I and III are both more stable than phase II. 
SERGIO E. MANGIONI AND JAVIER FERNANDEZ ACEVEDO

PHYSICAL REVIEW E 98, 052213 (2018)

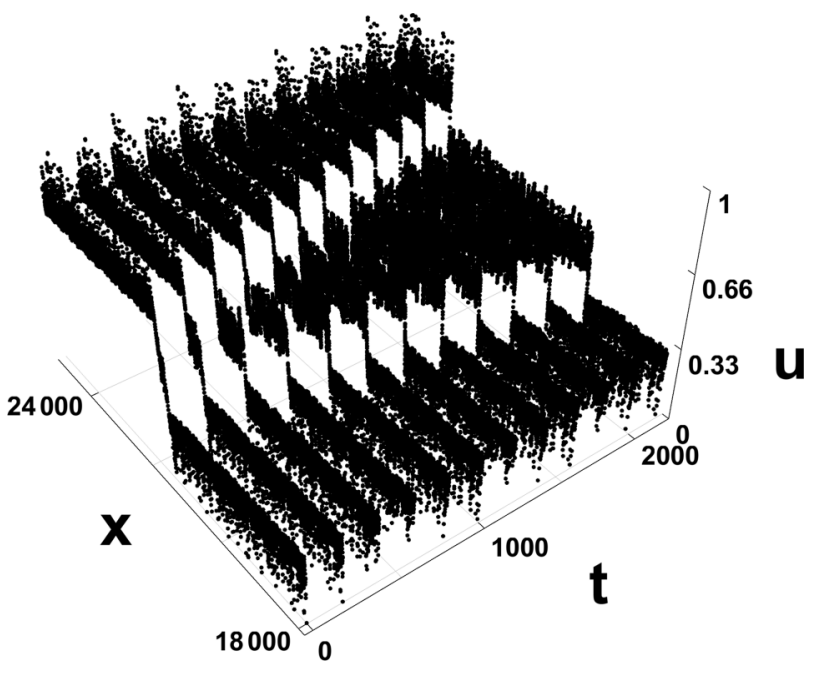

(a)

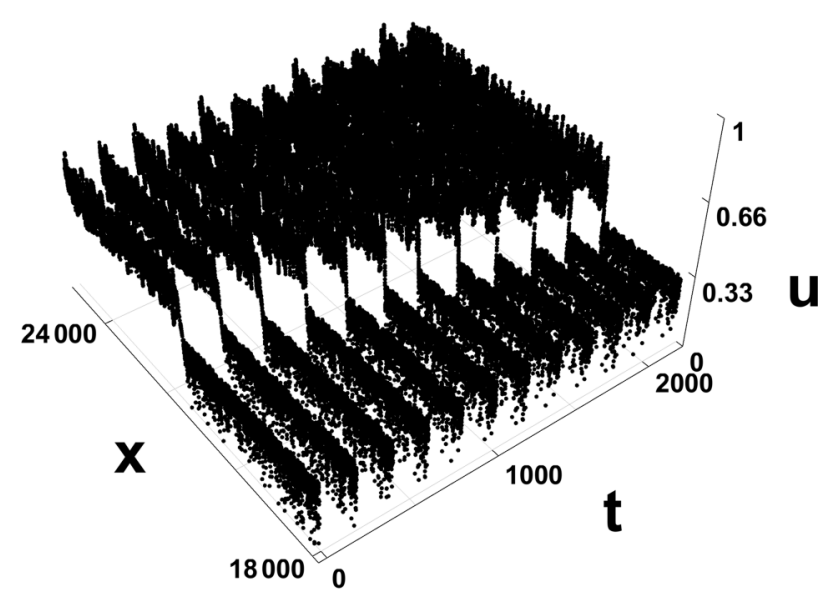

(b)

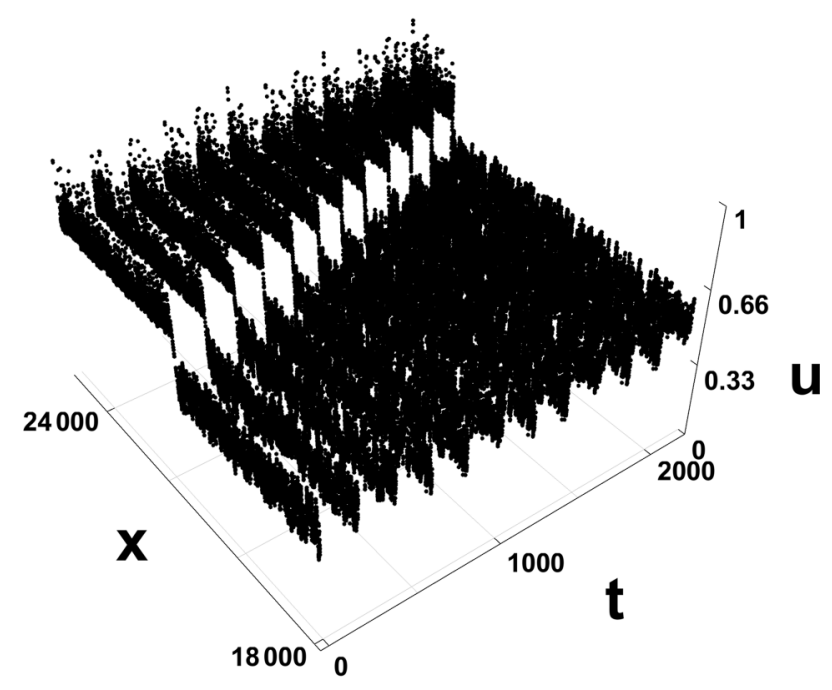

(c)

FIG. 4. Fronts for $\alpha$ fluctuations around $\alpha_{0}=0.5$, with $\beta_{d}=$ $0.333333, \beta_{u}=0.6666667, \lambda=1.7$, and $K_{a}=1$, when initiating the system from hyperbolic-tangent-like profiles connecting field values of different regions. (a) $\mapsto$ case (a): I-III. (b) $\mapsto$ case (b): I-IIu. (c) $\mapsto$ case (c): IId-III.

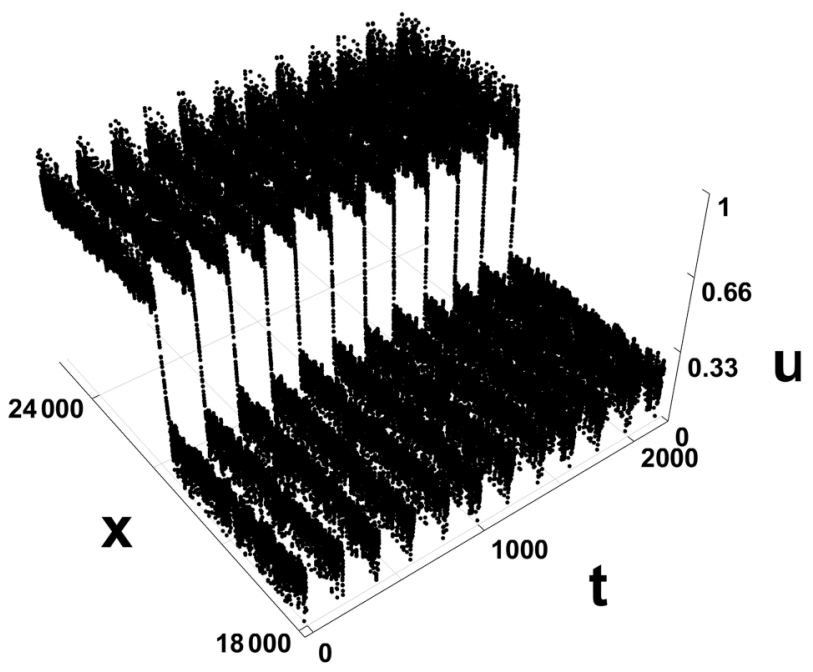

(a)

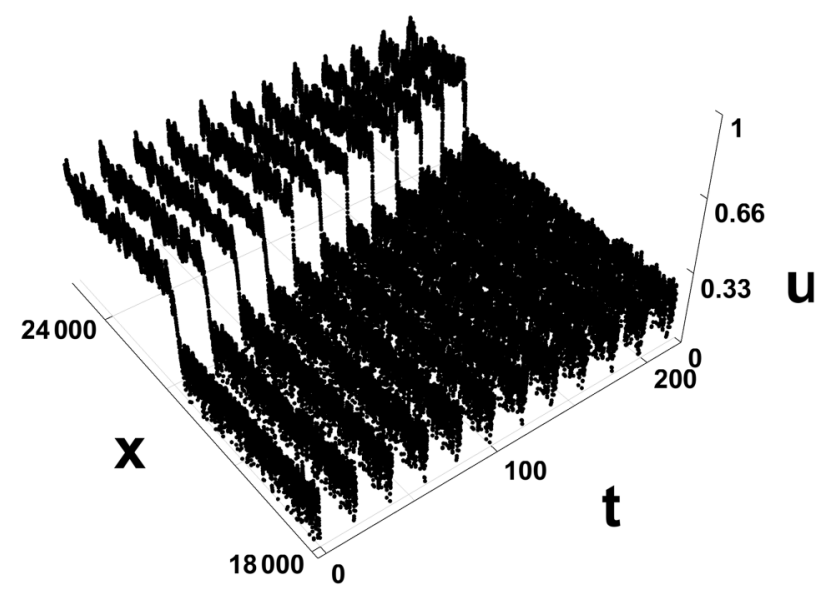

(b)

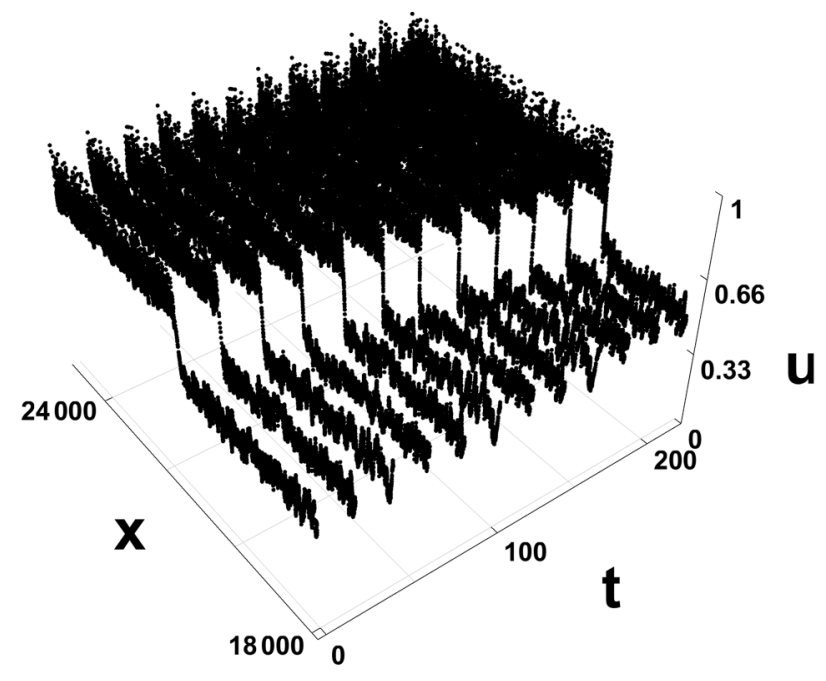

(c)

FIG. 5. Fronts for $\alpha$ fluctuations around $\alpha_{0}=0.5$, with $\beta_{d}=$ $0.333333, \beta_{u}=0.6666667, \lambda=1.7$, and $K_{a}=5$., when initiating the system from hyperbolic-tangent-like profiles connecting field values of different regions. (a) $\mapsto$ case (a): I-III. (b) $\mapsto$ case (b): I-IIu. (c) $\mapsto$ case (c): IId-III.

052213-6 


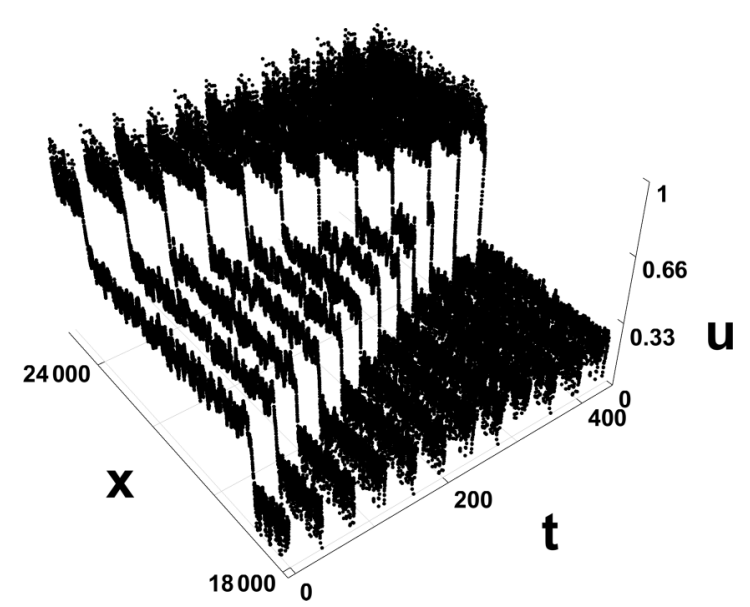

FIG. 6. Fronts for $\alpha$ fluctuations around $\alpha_{0}=0.5$, with $\beta_{d}=$ $0.333333, \beta_{u}=0.6666667, \lambda=1.7$, and $K_{a}=5$, when initiating the system from a profile with double fronts as the ones shown in the top panel of Fig. 4. Each phase belongs to a different region. We note that these fronts move with opposite velocity to those corresponding to the case with $K_{a}=1$.

According to the logic of this behavior, if we initiate the system from a profile like those double fronts shown in top of the Fig. 4 (corresponding to $K_{a}=1$ ), but we set $K_{a}=5$, then both fronts (I-II and II-III) will reverse their velocity and they will move toward each other until they merge, becoming a unique front (I-III) in the process. We tested this prediction and we effectively obtained the expected result. Figure 6 shows the system evolution for this case.

\section{STRONG FLUCTUATIONS}

In this section we consider strong fluctuations of $\alpha$ and $\beta_{d}$. In particular, we study in detail situations with $\lambda=15$ when $\alpha$ fluctuates and $\lambda=10$ when $\beta_{d}$ fluctuates, and we focus on changes in front behavior when varying $K_{a}$. We would expect that for such high noise intensity the chance for front generation is null. However, we recorded fronts propagating under these conditions. The key to the issue lies in the fluctuation-free roots and both symmetry breakings, which result from a coupling-noise cooperation. These roots become thresholds which cannot be crossed by the field unless a field gradient connects both sides of them.

We note that situations in which the twin states next to the fluctuation-free roots are stabilized arise even if noise intensity is high. These take place when the coupling is weak enough, a fact that does not mean that the usual behavior can be recovered under such circumstance. Given the great noise intensity, the phases are so noisy that any threshold is largely overtaken by the field. But the field cannot overcome the fluctuation-free roots, and therefore, a phase transition between regions only occurs if it is driven by a field gradient between them (in other words, a front connecting such phases). Figure 7 shows fronts when $\alpha$ fluctuates for $K_{a}=0.1$ and $\lambda=10$. We remark that the field values are accumulated around the fluctuation-free roots though they never match them.

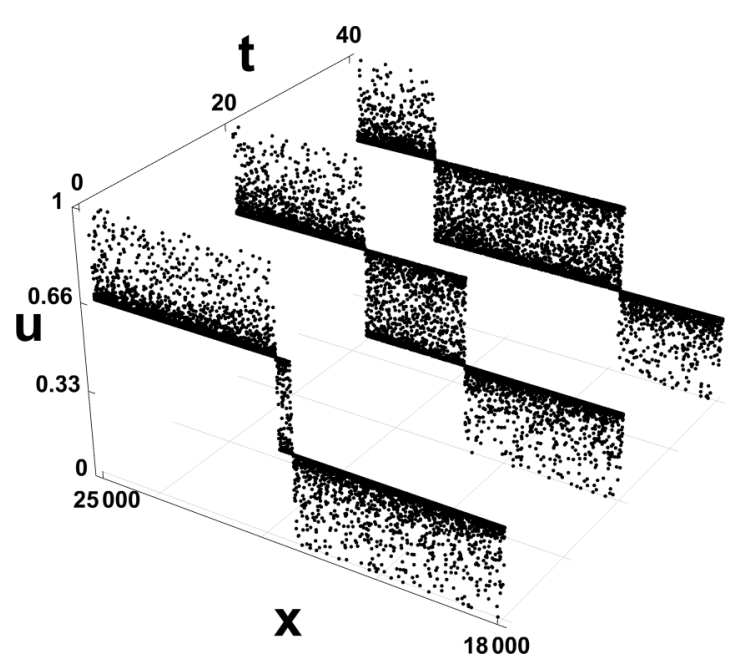

FIG. 7. Fronts for $\alpha$ fluctuations around $\alpha_{0}=0.5$, with $\beta_{d}=$ $0.333333, \beta_{u}=0.6666667, \lambda=10$, and $K_{a}=0.1$, when initiating the system from a profile I-III. We note that the field is accumulated around $\beta_{d}$ and $\beta_{u}$.

The results of the former section suggest that for large $\lambda$ values a change in coupling induces a change in the relation between phase stability corresponding to different regions. This would be the reason why the front velocity reverses itself when changing $K_{a}$. In other words, front behavior subject to high noise intensity could be governed by the "long-time dynamics," by which the criteria based on attraction basins unbalance (areas under the SPDF peaks) might be best suited to describe front behavior in such cases. We note that in this scenario the characterization of the dynamics as being of "long time" can be unclear. We consider the "long-time dynamics" to be the one that is set by the average stationary states of the system. Of course, such dynamics is one of long time compared to the evolution of the first moment of the field, but when noise intensity is strongly increased we expect the relaxation time to greatly decrease, to the point that the average stationary states are reached so quickly that the front dynamics ends up being dominated by such stationary states of the system. Our vision is that (a) for strong fluctuations, the field first accumulates around such states and a front connecting these states subsequently stabilizes and (b) for weak fluctuations, the field first accumulates around the states settled by the effective nonlinearity, given by the sum of the deterministic nonlinearity and the previously mentioned systematic part [Eq. (4)] and then a front that connects such states stabilizes before the average stationary states can be reached. Since for the strong fluctuations case the average stationary states are not only set by the nonlinearity parameters and noise effects but also by coupling (because of the symmetry breakings), we can understand why the $K_{a}$ value also impact on the direction of the front velocity.

Strong fluctuations of both $\alpha$ and $\beta_{d}$ produce the same results as those shown for $\lambda=1.7$ except phases are much noisier. In order to illustrate this situation, Fig. 8 displays cases with $\lambda=10$ for $\beta_{d}$ fluctuations with $K_{a}=0.56$, when initiating the system from profile I-III. We registered that the field belonging to each phase connecting the front is effectively confined within the corresponding region, and also, 


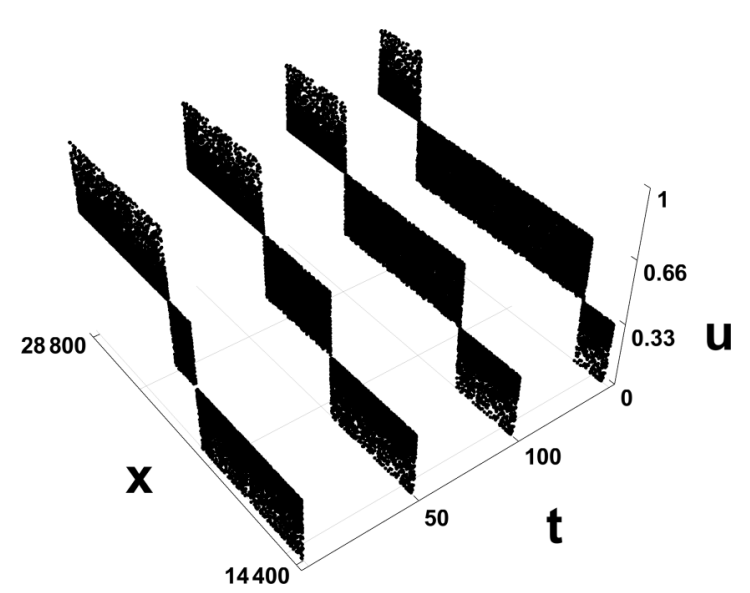

FIG. 8. Sample of different front types for $\beta_{d}$ fluctuations around $\beta_{d 0}=10^{-3}$ with $\alpha=0.33, \beta_{u}=0.66, \lambda=10$, and $K_{a}=0.56$. Initial condition: Profile I-III. The behavior is similar to the $\lambda=1.7$ case, with the exception that the field now takes values along its entire region of origin.

because of the great noise intensity, the field expands along all of its region. The observation of these fronts suggests their feasibility is supported by the existence of the "impassable walls" mentioned before. In this regard, fronts are rather connections between regions than connections between phases. For the sake of simplicity, hereinafter we will call the noisy phases connected by the fronts: $\phi^{\mathrm{I}}$ to the region I phase, $\phi^{\mathrm{II}}$ to the region II phase, and $\phi^{\mathrm{III}}$ to the region III phase.

In order to further characterize this phenomenon, we calculated the noisy front velocity $\left(V_{f}\right.$ measured in lattice sites per time step and calculated averaging on the basis of 30 different realizations) as a function of spatial coupling constant $K_{a}$ (since the unusual behavior arises by varying $K_{a}$ we choose this parameter instead of $\lambda$ ). When $\alpha$ fluctuates, we only consider a situation similar to the former quasisymmetric one (region sizes are almost equal): $\beta_{u}=0.66$ (slightly less than $\frac{2}{3}$ ), $\beta_{d}=0.33$ (slightly less than $\frac{1}{3}$ ), and $\alpha_{0}=0.5$. In order to avoid mix-ups regarding the sign of front velocity we made it clear that $V_{f}>0$ provided that front I-II propagates toward region II (remaining $\phi^{\mathrm{I}}$ ), front II-III propagates toward region III (remaining $\phi^{\mathrm{II}}$ ), and front I-III propagates toward region III (remaining $\phi^{\mathrm{I}}$ ). Figure 9 shows the corresponding curves. The left graphic shows the cases when the system is initiated from profiles I-II and II-III, and the right graphic shows the case when the system is initiated from profile I-III. We note that noisy fronts I-III are only stabilized when $K_{a}$ exceeds a critical value we named $K_{a}^{c}$. When observing the curves in the left graphic we see that the fronts I-II and II-III can be stabilized for $K_{a}$ values beyond $K_{a}^{c}$, but we know those fronts will propagate toward each other until they merge in a front I-III. Concerning fronts I-II and II-III, we note that the $K_{a}$ values for which the front velocity reversion occurs are slightly different. A choice of perfectly symmetric parameters eliminates such a difference and produces fronts I-III at rest.

When $\beta_{d}$ fluctuates, we examine quasisymmetric and asymmetric situations. We do not show here the corresponding curves for the quasisymmetric case, since these are very

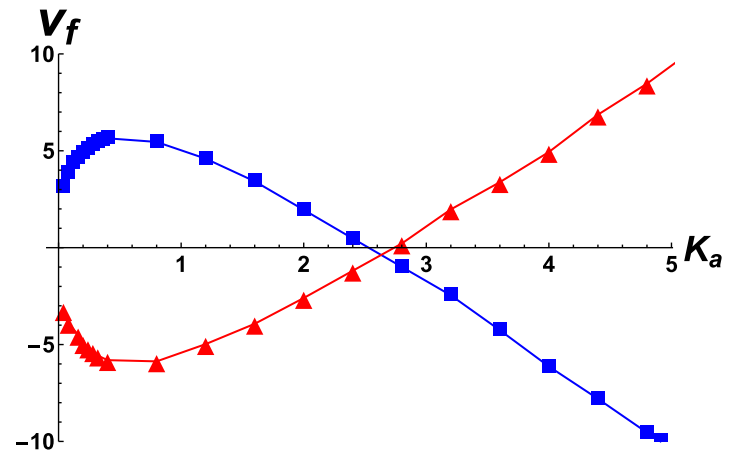

(a)

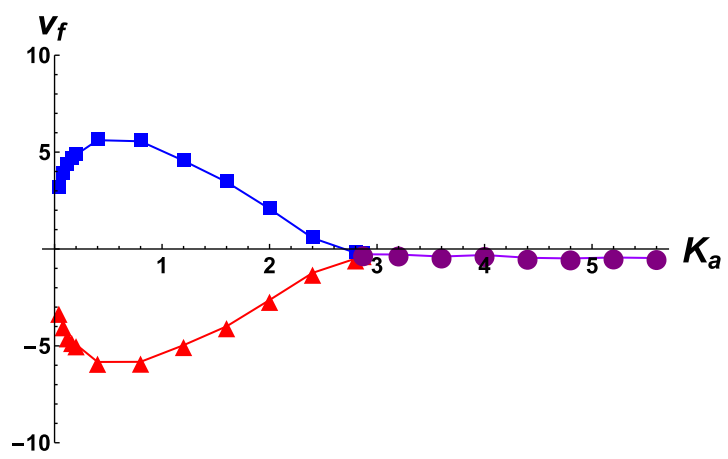

(b)

FIG. 9. Noisy front average velocity $\left(V_{f}\right)$ vs. spatial coupling constant $K_{a}$ for $\alpha$ fluctuations around $\alpha_{0}=0.5$ with $\beta_{d}=0.33$, $\beta_{u}=0.66$, and $\lambda=15$. (a) $\mapsto$ fronts I-II when initiating the system from profiles I-II $(\boldsymbol{\Delta})$ and fronts II-III when initiating the system from profiles II-III (ם). The velocity reversion points of fronts I-II and II-III by varying $K_{a}$ are clearly identified with the "zeros" of such curves. Since this situation is not completely symmetrical, $V_{f}$ is slightly different from zero where the curves intersect $\left(K_{a}^{c} \simeq 2.87\right)$. (b) $\mapsto$ fronts I-II $(\boldsymbol{\Delta})$, fronts II-III (ם), and fronts I-III $(\bullet)$, where the system in the three cases is initiated from profiles I-III. We note that the noisy fronts I-II and II-III can be obtained from initial profiles I-II and II-III but also whenever $K_{a}<K_{a}^{c}$ from initial profiles I-III. Regardless of the initial condition, the $V_{f}$ values calculated for each $K_{a}$ value are the same. When the initial condition is a profile I-III, noisy fronts I-III are only obtained for $K_{a}>K_{a}^{c}$. It is evident that the noisy fronts I-II and II-III are more stable than noisy fronts I-III for $K_{a}<K_{a}^{c}$.

similar to the above case. The asymmetry is introduced in the region sizes by changing the parameter values characterizing the nonlinearity. Figure 10 shows the velocity curves for $\beta_{u}=$ $0.9, \alpha=0.45$, and $\beta_{d 0}=10^{-3}$ (see Supplemental Material at [17] for an intermediate case). We observe that $K_{a}^{c}$ values increases as the system asymmetry increases. We note as well that, for larger $\alpha$ and $\beta_{u}$ values, the overall view of the slopes of such curves becomes pronouncedly positive, which means that, in this case, all noisy fronts tend to move in the same direction, even though their velocities remain different.

Since we think that the long-time dynamics strongly influences the behavior of these fronts, we calculated the corresponding SPDF $\left(P_{\mathrm{st}}\right)$ in order to look for any hint about its relation with the propagation direction. We do not expect such simple thing as $V_{f} \propto-\left.\ln \left[P_{\text {st }}\right]\right|_{\phi^{\text {ms }}} ^{\phi^{s}}$ (Maxwell's area law applied to the effective potential, $\phi^{s}$ indicates stable phase 


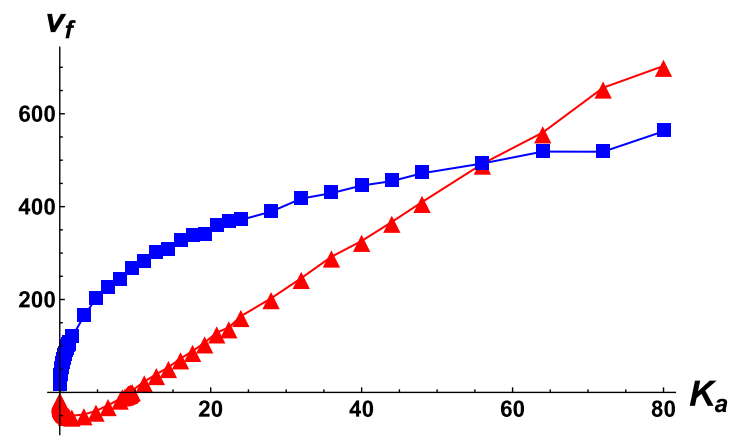

(a)

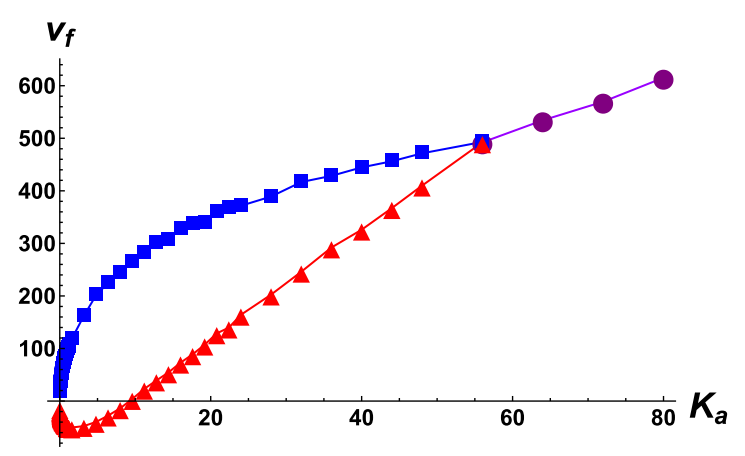

(b)

FIG. 10. Noisy front average velocity $\left(V_{f}\right)$ vs. spatial coupling constant $K_{a}$ for $\beta_{d}$ fluctuations around $\beta_{d 0}=10^{-3}$, with $\alpha=0.45$, $\beta_{u}=0.9$, and $\lambda=10$. (a) $\mapsto$ fronts I-II when initiating the system from profiles I-II $(\boldsymbol{\Delta})$ and fronts II-III when initiating the system from profiles II-III (ם). The overall slope has increased so much that only front II-III reverse its velocity ( $K_{a}^{c} \simeq 56$ corresponding to $V_{f} \simeq 493$ ). (b) $\mapsto$ fronts I-II ( $\Delta$ ), fronts II-III (ם), and fronts I-III $(\bullet)$, where the system in the three cases is initiated from profiles IIII. We note that the overall slope increasing with the asymmetry in region sizes.

and $\phi^{\mathrm{ms}}$ indicates metastable phase), but we do expect that at least such intense noise drives the fronts to promote phase transitions toward the most probable average state.

We consider appropiate to discuss about "the most probable average state." Such a state could be taken to be the higher SPDF peak. This definition, which is suitable for additive noise and even for multiplicative noise when its factor is never zero, could be suggested based on Maxwell's area law. The SPDF peaks are narrow for low noise intensity, and therefore, it can be expected that the higher peak is also the one of larger area or at least that the difference between these situations is insignificant. However, such a relation could change for high noise intensity. In fact, wide and low peaks of SPDF that compete with narrow and high peaks of SPDF could arise. What is "the most probable average state" in such a case? We are describing noisy states, and therefore we consider the area under each peak as the relevant variable defining the issue. The larger area peak expresses "the most probable average state" for us. So, we associate the attraction basins with the area under each peak.

This time, in order to calculate the SPDF, the system was initiated from a uniform random distribution between $u=0$ and $u=1$ in order to enable the field to flow between regions

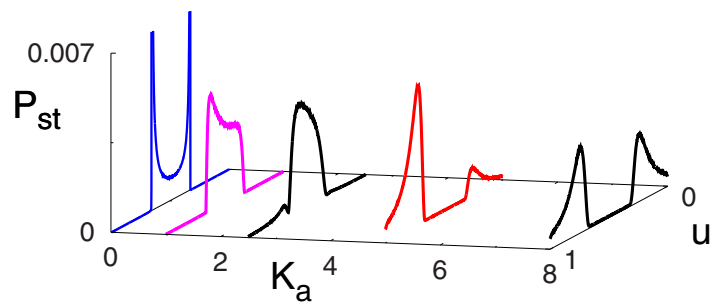

FIG. 11. Numerically computed SPDF when initiating the system from a uniform random distribution between $u=0$ and $u=1$ : $P_{\text {st }}$ vs. $u$ for $\beta_{d}$ fluctuations around $\beta_{d 0}=10^{-3}$, with $\alpha=0.33$, $\beta_{u}=0.66, \lambda=10$, and $K_{a}$ values between 0 and 8 . Reversions of attraction-basin sizes are observed between regions I-II and II-III.

and, therefore, the competition between its corresponding states. We ensured a good statistical average by initiating the system from 30 different seeds. Figure 11 shows the results for a $K_{a}$ value range including the reversion points of the front velocity (fluctuations of $\beta_{d}$ around $\beta_{d 0}=10^{-3}$, with $\alpha=0.33, \beta_{u}=0.66$, and $\lambda=10$ ). When comparing it with Fig. 9, we observe that the reversions of the attraction-basin relation is linked to velocity reversions of fronts I-II and II-III, which is consistent with the fact that fronts propagate in a way that promotes the most stable average phase. In other words, this result suggests that the long-time dynamics governs the behavior of these fronts. This link between the attraction-basin relation and the front propagation direction is fulfilled by all curves shown in Fig. 11. However, close enough to the reversion point such a link is lost. We observed a small difference between the $K_{a}^{t}$ corresponding to the velocity reversion and the $K_{a}^{e}$ corresponding to reversion of the attraction-basin relation, which points out the presence of another minor effect influencing the front building process (see an extended version at Supplemantal Material [17]). However, numerical results point out that the dominating effect setting the front propagation direction is the unbalance between attraction basins established in the long timescale, promoting the state of minimum effective potential. The front moves in such way that always the most probable state is set, except for $K_{a}$ values within the aforementioned range. Of course, we also obtained the same results for $\alpha$ fluctuations and other parameter values provided that noise intensity is high enough.

\section{ANALYSIS AND CONCLUSIONS}

We have worked here with a nonlinearity habitually used to illustrate the general behavior of fronts when one of the characterizing parameters fluctuates. As long as the noise intensity is below a certain threshold, the average front behavior is suitably described by changing the deterministic nonlinearity to another one that adds an identical term to that of Stratonovich's. Given that this nonlinearity (deterministic nonlinearity plus the systematic part) is the same as that used to support the so-called short-time instability, we understand that the front is built before the average stationary state (state describing the system in the long timescale) is reached and then it propagates toward a direction dictated by such nonlinearity. Therefore, the noise first promotes the noisier state by 
means of front propagation (we numerically observed such a phenomenon), but a long time after this state is conformed, the same noise starts to push the system toward the lessnoisy state, because this is its most favored one in the long timescale [such as what is set by Eq. (3)]. So, below threshold, whenever the attraction basins of the deterministic part are balanced, the average state favored by the front propagation is also the one less probable in the long-time regime (the noisier one). We remark that under these conditions, the front propagation direction is independent of the coupling constant.

In this work, we have shown that the front behavior is very different when noise intensity is much above said threshold. The ingredients supporting this behavioral change are several. First, the nonlinearity roots split the field variability range into three regions, working as impassable walls that prevent the field from crossing them, unless a field gradient connecting those regions exists. Second, when noise intensity overcomes the above-mentioned threshold, a coupling-noise cooperation causes two symmetry breakings of the fluctuation-free stable states, which are enabled by a short-time instability. As a consequence, as long as the noise intensity is high enough, three new stable average states emerge, which we identified with the SPDF peaks, each of them confined within its own region. We note that under such conditions the field expands in all the confinement region. Third, although the symmetry breakings are originated because of an instability in the short timescale (which sets the threshold), these three new average states (which we identify with SPDF peaks) are generated in the long timescale and their localization and general characteristics depend heavily on coupling; so much so that a reversion of the area relation (as well as of the height relation) of the SPDF peaks occurs by changing the $K_{a}$ value. Fourth, the noise promotes the less-noisy state in the long timescale. Fifth, the front propagation direction depends on the coupling constant, in fact, a velocity reversion is produced for a given $K_{a}$ value. Sixth, fronts propagate in the direction favoring the most probable average state (largest area peak which also matches the heighest peak of SPDF), except for a small range of $K_{a}$ values around the reversion point. We note that this is an opposite behavior to when noise intensity is below threshold. More precisely, the propagation direction is correlated with the area-height relation between probability peaks (gap between the attraction basins) and the corresponding reversion points are produced for close $K_{a}$ values.

The sum of these ingredients leads us to conclude that when noise intensity is quite superior to the threshold value, the average fronts happen between very noisy phases, each one fully expanding inside its corresponding region. These phases, the result of long-time dynamics modified by a shorttime instability, set out preferably the average-front propagation direction. Since the area-height relation between probability peaks that correspond to these noisy phases depends heavily on the coupling constant and it is correlated with the average-front propagation direction, we infer that the long-time dynamics is what impacts the most on the average behavior of such fronts. We highlight that, a reversion of both the front propagation direction and area-height relation between probability peaks happens by changing the $K_{a}$ value. These reversion points are not entirely matching; however, the difference between them is small compared to the $K_{a}$ values range involving the phenomenon.

In conclusion, we showed that there is a threshold for noise intensity far beyond which front behavior is very different to the cases with low noise intensity. Resorting to numerical calculation, we identified three highly noisy phases and observed three different types of fronts. Moreover, we observed that situations involving the three phases simultaneously, in which two fronts move away from each other, can be reverted by changing the coupling constant. Finally, we found an explanation supporting such a phenomenon.

\section{ACKNOWLEDGMENTS}

Financial support from Consejo Nacional de Investigaciones Científicas y Técnicas (CONICET) and Universidad Nacional de Mar del Plata (UNMdP) of Argentina is acknowledged.

\section{APPENDIX}

The equation describing the long-time behavior can be obtained by using the so-called entropic mechanism. Following the original proposal of Ibañes et al. [6], we mapped the system into a nongradient relaxational one by rewriting Eq. (1) as:

$$
\partial_{t} u=-\Gamma(u) \frac{F_{0}(u)+D \partial_{x}^{2} u}{-\Gamma(u)}+\Gamma^{1 / 2}(u) \eta(x, t) .
$$

Subsequently, we define a free energy functional $\mathcal{F}[u(x)]$ so that:

$$
\frac{\delta \mathcal{F}[u(x)]}{\delta u(x)}=\frac{F_{0}(u)+D \partial_{x}^{2} u}{-\Gamma(u)},
$$

and therefore Eq. (3) can be written as:

$$
\partial_{t} u=-\Gamma(u) \frac{\delta \mathcal{F}[u]}{\delta u(x)}+\Gamma^{1 / 2}(u) \eta(x, t) .
$$

Under these conditions, the corresponding $\operatorname{SPDF} P_{\mathrm{st}}(u)$ is of Boltzmann's type [6,7]:

$$
P_{\mathrm{st}}(u) \propto \exp \left\{-\frac{2 \mathcal{F}_{\mathrm{eff}}[u]}{\bar{\lambda}^{2}}\right\}
$$

in terms of an effective free-energy functional:

$$
\mathcal{F}_{\text {eff }}[u]=\mathcal{F}[u]+\frac{\sigma^{2}}{4} \int_{-L}^{L} \mathrm{~d} x \ln [\Gamma(u)],
$$

Fortunately, the long-time average dynamics driven by this noise can be studied without explicit knowledge of the functional $\mathcal{F}[u(x)][18-21]$. It suffices to replace $\mathcal{F}_{\text {eff }}[u(x)]$ by $\mathcal{F}[u(x)]$ in Eq. (A3) and remove the noise term in order to retrieve Eq. (3), which describes the long-time average dynamics. 
[1] J. D. Murray, Mathematical Biology (Springer, New York, 1993).

[2] A. Engel, Noise-induced front propagation in a bistable system, Phys. Lett. A 113, 139 (1985).

[3] J. García-Ojalvo and J. M. Sancho, Noise in Spatially Extended System (Springer, New York, 2012).

[4] C. Van den Broeck, J. M. R. Parrondo, and R. Toral, NoiseInduced Nonequilibrium Phase Transition, Phys. Rev. Lett. 73, 3395 (1994).

[5] C. Van den Broeck, J. M. R. Parrondo, R. Toral, and R. Kawai, Noneequilibrium phase transitions induced by multiplicative noise, Phys. Rev. E 55, 4084 (1997).

[6] M. Ibañes, J. García-Ojalvo, R. Toral, and J. M. Sancho, NoiseInduced Scenario for Inverted Phase Diagrams, Phys. Rev. Lett. 87, 020601 (2001).

[7] O. Carrillo, M. Ibañes, J. García-Ojalvo, J. Casademunt, and J. M. Sancho, Intrinsic noise-induced phase transitions: Beyond the noise interpretation, Phys. Rev. E 67, 046110 (2003).

[8] S. E. Mangioni and R. R. Deza, Stochastic dissipative solitons, Phys. Rev. E 92, 032116 (2015).

[9] J. Armero, J. M. Sancho, J. Casademunt, A. M. Lacasta, L. Ram'rrez-Piscina, and F. Sagués, External Fluctuations in Front Propagation, Phys. Rev. Lett. 76, 3045 (1996).

[10] D. Panja, Effects of fluctuations on propagating fronts, Phys. Rep. 393, 87 (2004).

[11] Our tests using low noise intensity (numerical simulations) proved to be consistent with the second approach in all the situations outlined by us, even for the lowest noise intensity values we considered.

[12] L. A. da Silva, E. H. Colombo, and C. Anteneodo, Effect of environment fluctuations on pattern formation of single species, Phys. Rev. E 90, 012813 (2014).
[13] C. Gabaldón, J. Montero-Pau, C. M. J, and M. Serra, Lifehistory variation, environmental fluctuations and competition in ecologically similar species: modeling the case of rotifers, J. Plankton Res. 37, 953 (2015).

[14] S. Mangioni, Generalized Nagumo model: Interesting effects of parameters fluctuations, Eur. Phys. J. B 88, 265 (2015).

[15] S. E. Mangioni, Cooperation between fluctuations and spatial coupling for two symmetry breakings in a gradient system, Phys. Rev. E 92, 062136 (2015).

[16] S. R. Carpenter, D. Ludwig, and W. A. Brock, Management of eutrophication for lakes subject to potentially irreversible change, Ecol. Appl. 9, 751 (1999).

[17] See Supplemental Material at http://link.aps.org/supplemental/ 10.1103/PhysRevE.98.052213 for a) For when fronts are initiated from profiles I-IId, or IIu-III, and around critical noise intensity. In particular, infinitisimal fronts connecting these regions are stabilized. Moreover, When fronts are initiated from a profile I-III, such fronts arise within the same front I-III. These results are consistent with the existence of the "impassable walls". b) For a more detailed version of results regarding the strong fluctuations regime.

[18] S. E. Mangioni and H. S. Wio, Interplay between noise and boundary conditions in pattern formation in adsorbed substances, Phys. Rev. E 71, 056203 (2005).

[19] S. E. Mangioni, Nano-pattern stabilization by multiplicative noise, Physica A 389, 1799 (2010).

[20] S. E. Mangioni and R. R. Deza, Nonequilibrium pattern formation by subdominant attractive forces: A simple model and a stabilization strategy by means of noise, Phys. Rev. E 82, 042101 (2010).

[21] S. E. Mangioni and R. R. Deza, Stabilizing transient nanopatterns in heterogeneous catalysis: A comprehensive explanation of the phenomenon, Physica A 391, 4191 (2012). 